\title{
Graph Grammar Based Petri Nets Model of Concurrency for Self-adaptive $h p$-Finite Element Method with Triangular Elements
}

\author{
Arkadiusz Szymczak and Maciej Paszyński \\ Department of Computer Science \\ AGH University of Science and Technology, \\ Al. Mickiewicza 30, 30-059 Cracow, Poland \\ arek.szymczak@gmail.com, paszynsk@agh.edu.pl \\ http://home.agh.edu.pl/ paszynsk
}

\begin{abstract}
The paper presents the model of concurrency for the selfadaptive $h p$-Finite Element Method ( $h p$-FEM) with triangular elements. The model concerns the process of an initial mesh generation as well as mesh adaptation. The model is obtained by defining CP-graph grammar productions as basic undivided tasks for both mesh generation and adaptation algorithms. The order of execution of graph grammar productions is set by control diagrams. Finally, the Petri nets are created based on the control diagrams. The self-adaptive $h p$-FEM algorithm modeled as a Petri net can be analyzed for deadlocks, starvation or infinite execution.
\end{abstract}

\section{Introduction}

In this paper we present the model of concurrency for the self-adaptive $h p$ Finite Element Method ( $h p$-FEM) with triangular elements. The self-adaptive $h p$-FEM algorithm starts from an arbitrary initial mesh and generates a sequence of meshes delivering exponential convergence of the numerical error with respect to the mesh size 415. This is done by breaking selected elements into smaller elements (this procedure is called $h$ refinements) or by modifying the polynomial order of approximation on selected element edges or interiors (this procedure is called $p$ refinement). The algorithm can be utilized for solving two and three dimensional engineering problems with very high accuracy [45].

The parallel version of both two and three dimensional self-adaptive $h p$-FEM algorithms were created according to the domain decomposition paradigm 667. The parallel implementations, although efficient and scalable, showed the need for further theoretical analysis of the concurrency hidden within the self-adaptive algorithm. Two graph grammar models for both sequential and parallel $h p$ FEM algorithms were created, for both rectangular [8] and triangular [9] finite elements. The models utilize the $\mathrm{CP}$ graph grammar introduced by [1/2]3].

In this paper, we extend the graph grammar model presented in [9] for the case of generation of an arbitrary initial mesh. We also create the model of concurrency, expressed by control diagrams setting the order of execution of 
graph grammar productions, and presenting which productions can be executed concurrently. Finally, the Petri nets are created, for both initial mesh generation and mesh adaptation algorithms. The Petri nets model will allow for creating the reachability graphs, finding place/transition invariants, and similar tools for the self-adaptive $h p$-FEM algorithm. The $h p$-FEM modeled as a Petri net can be analyzed for deadlocks, starvation or infinite execution.

\section{2D Mesh Generation}

In this section we present a set of graph grammar productions for generation of two-dimensional mesh with triangular finite elements. This extends the graph grammar introduced in [9] generating a linear sequence of triangular elements. The new productions PI1 - PI8, presented in Figure 1, realize the topology generation of an arbitrary shape $2 \mathrm{D}$ triangular mesh. Meaning of the node symbols is as follows:

- E1, E2 - type-1 (triangle) and type-2 (upside-down triangle) element, respectively

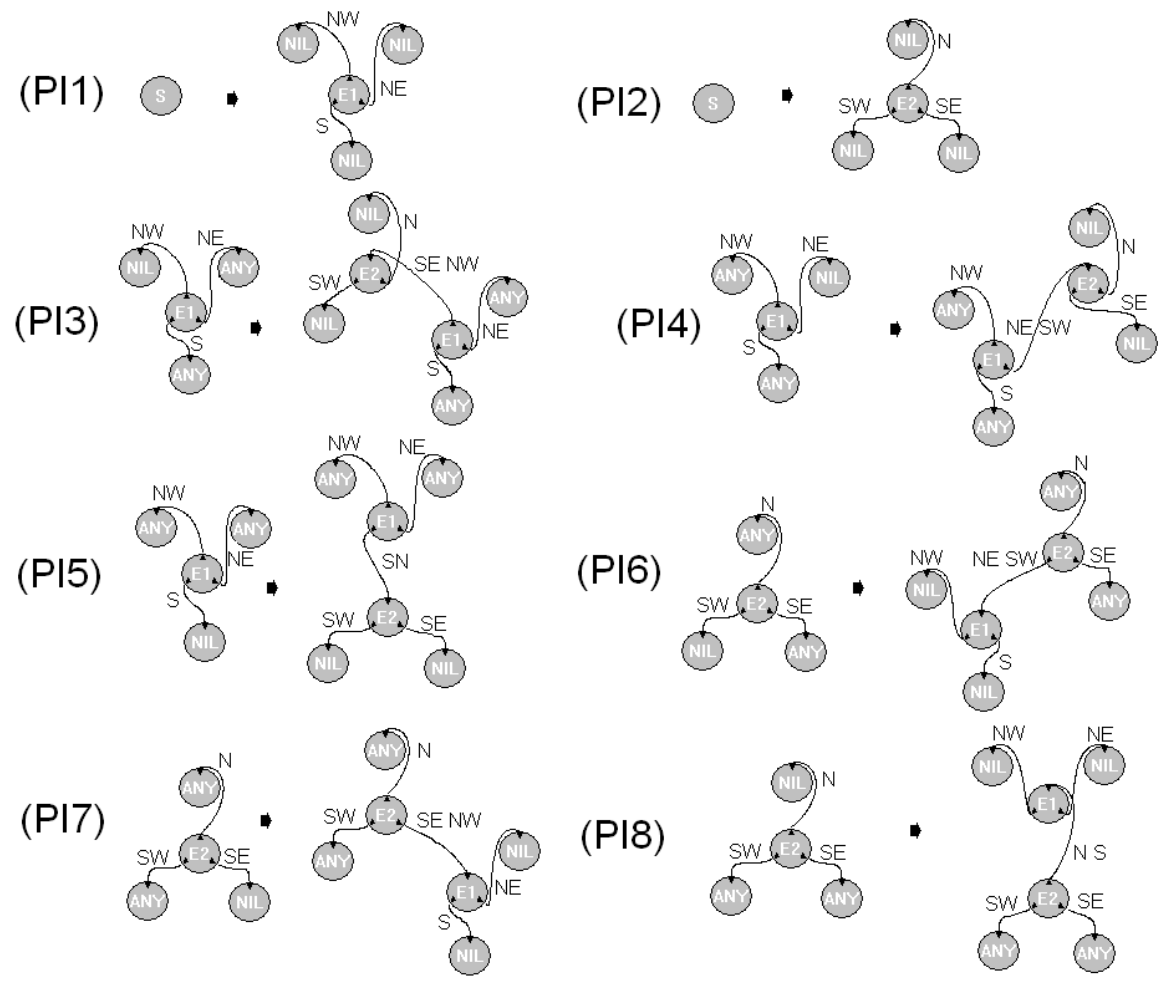

Fig. 1. Set of graph grammar productions PI1 - PI8 for the generations of triangular finite element two dimensional mesh 
(PI9)
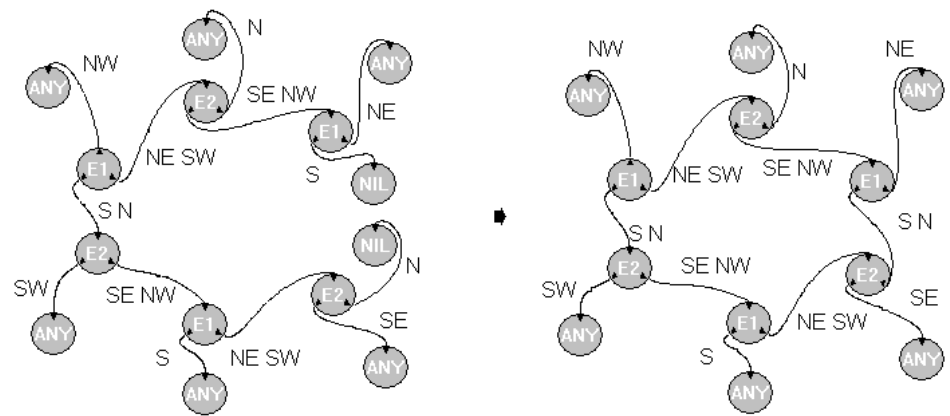

(PI10)
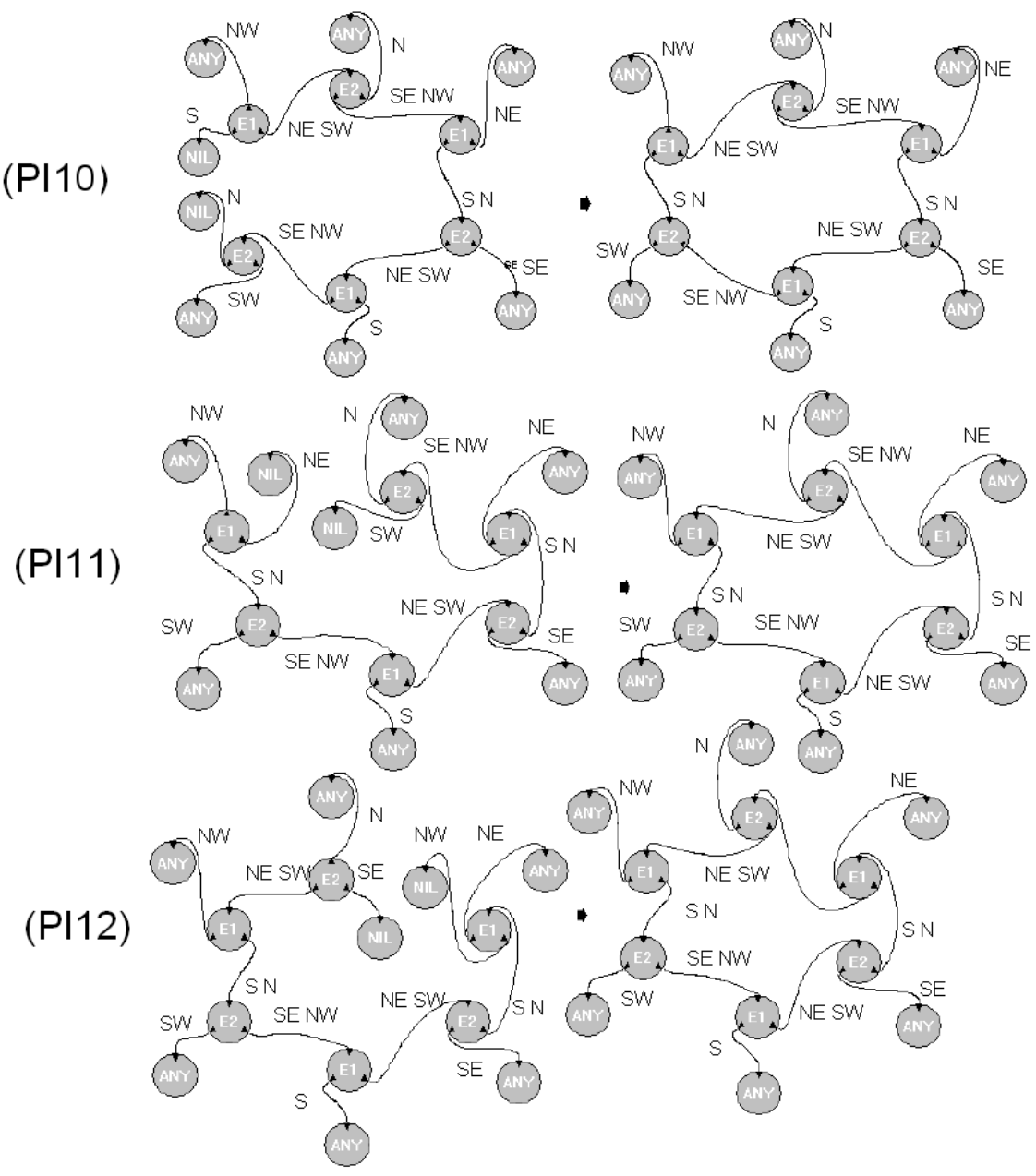

Fig. 2. Graph grammar productions PI9 - PI12 connecting layers of generated elements 
- NIL - fake node denoting no adjacent node

- $A N Y$ - fake node meaning that given node is irrelevant to the given production

The new productions PI9 - PI14, presented in Figure 2 and 3, are responsible for identification of adjacent elements to prevent element overlapping. Since new element is always derived from one existing element and each element can have up to 3 adjacent elements, missing up to 2 bonds must be detected. Therefore, as soon as any of productions PI9 - PI14 matches, it must be executed before subsequent execution of productions PI1 - PI8.

We summarize this section with an exemplary derivation of a single row of triangular finite element mesh. The mesh is generated by the following sequence of graph grammar productions: PI1 - PI4 - PI7 - PI4. The resulting graph and the corresponding finite element mesh is presented in Figure 4.

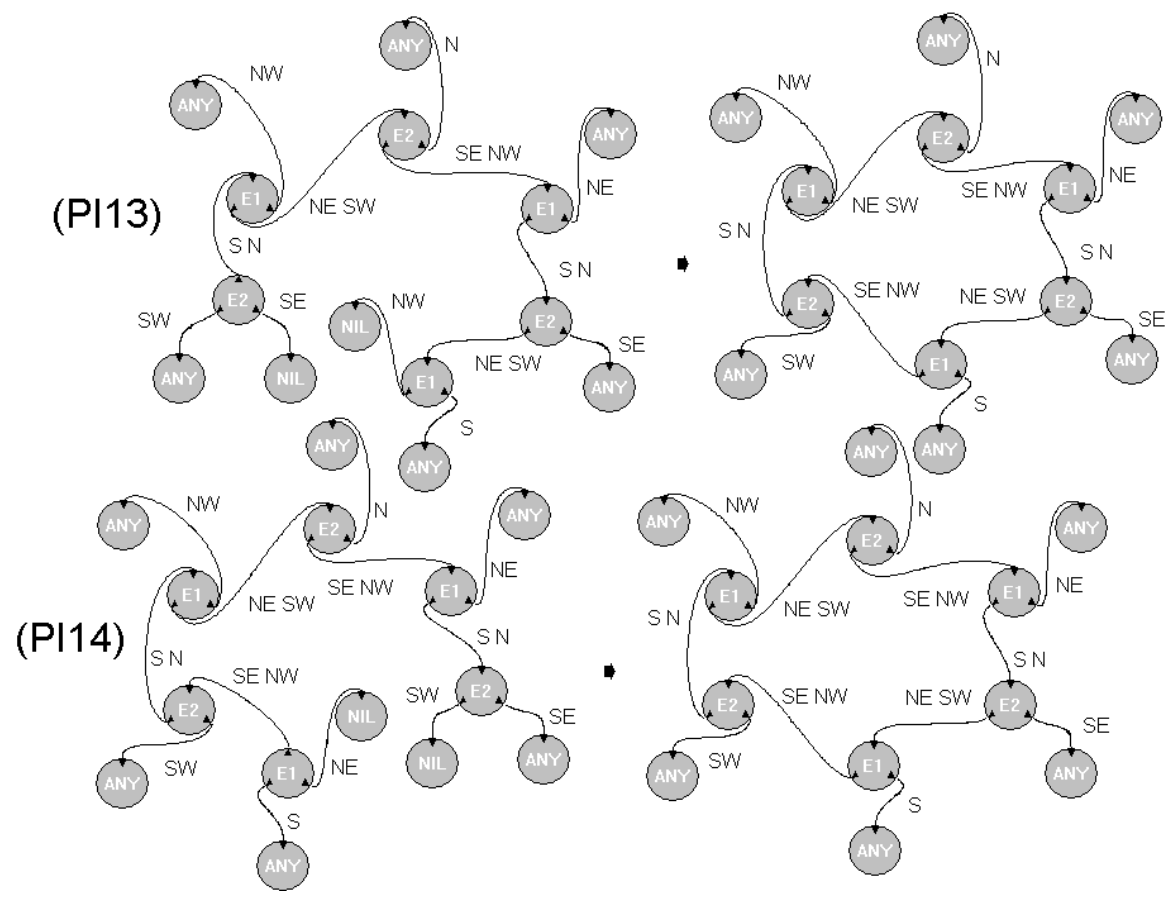

Fig. 3. Graph grammar productions PI13 - PI14 connecting layers of generated elements

\section{Graph Grammar Based Model of Concurrency}

In this section we present the control diagram based model of concurrency for the process of an initial mesh generation as well as mesh $h$ adaptation. The model 

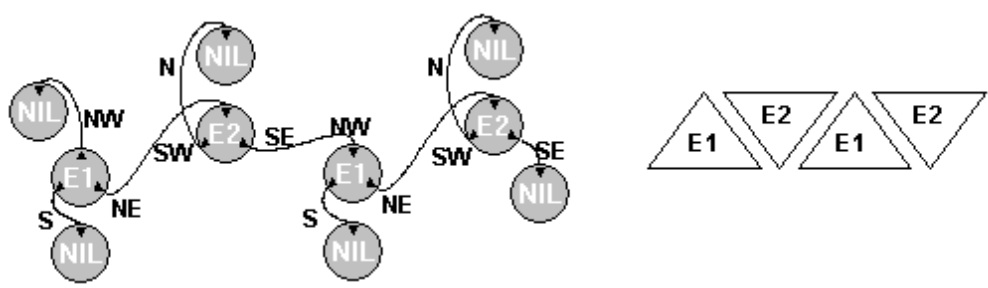

Fig. 4. Exemplary finite element mesh obtained by executing the following sequence of graph grammar productions PI1 - PI4 - PI7 - PI4

is presented in Figure 5. The following list summarized symbols used in the control diagrams. Some graph grammar productions referred in the list were defined in 8], while the productions PI1 - PI14 were described in the previous section.

- PE1 - production for generation of type 1 element structure

- PE2 - production for generation of type 2 element structure

- $P C E H$ - production for identification of common edge of horizontally adjacent elements

- $P C E V$ - production for identification of common edge of vertically adjacent elements

- PJI1 - production allowing for breaking type 1 element interior

- PJI2 - production allowing for breaking type 2 element interior

- PFE - production allowing for breaking edge between two broken interiors

- PBI1 - production for breaking type 1 element interior

- PBI2 - production for breaking type 2 element interior

- PBE - production for breaking element edge

- PWest1 - production for propagation of adjacency data for west edge of element type 1

- PEast1 - production for propagation of adjacency data for east edge of element type 1

- PSouth - production for propagation of adjacency data for south edge of element type 1

- PWest2 - production for propagation of adjacency data for west edge of element type 2

- PNorth - production for propagation of adjacency data for north edge of element type 2

- PEast2 - production for propagation of adjacency data for east edge of element type 2

The generation of an initial mesh can be highly parallelized, as it is illustrated on the left panel in Figure 5. While subsequent elements are being added to the mesh, the structure of already existing elements may be constructed and their common edges may be identified. Adaptation of the mesh is more sequential, as it is presented on the right panel in Figure 5. Generally, while all possible transformations of the same type can be executed concurrently, different types of transformations must be executed in the predefined order. However, since 

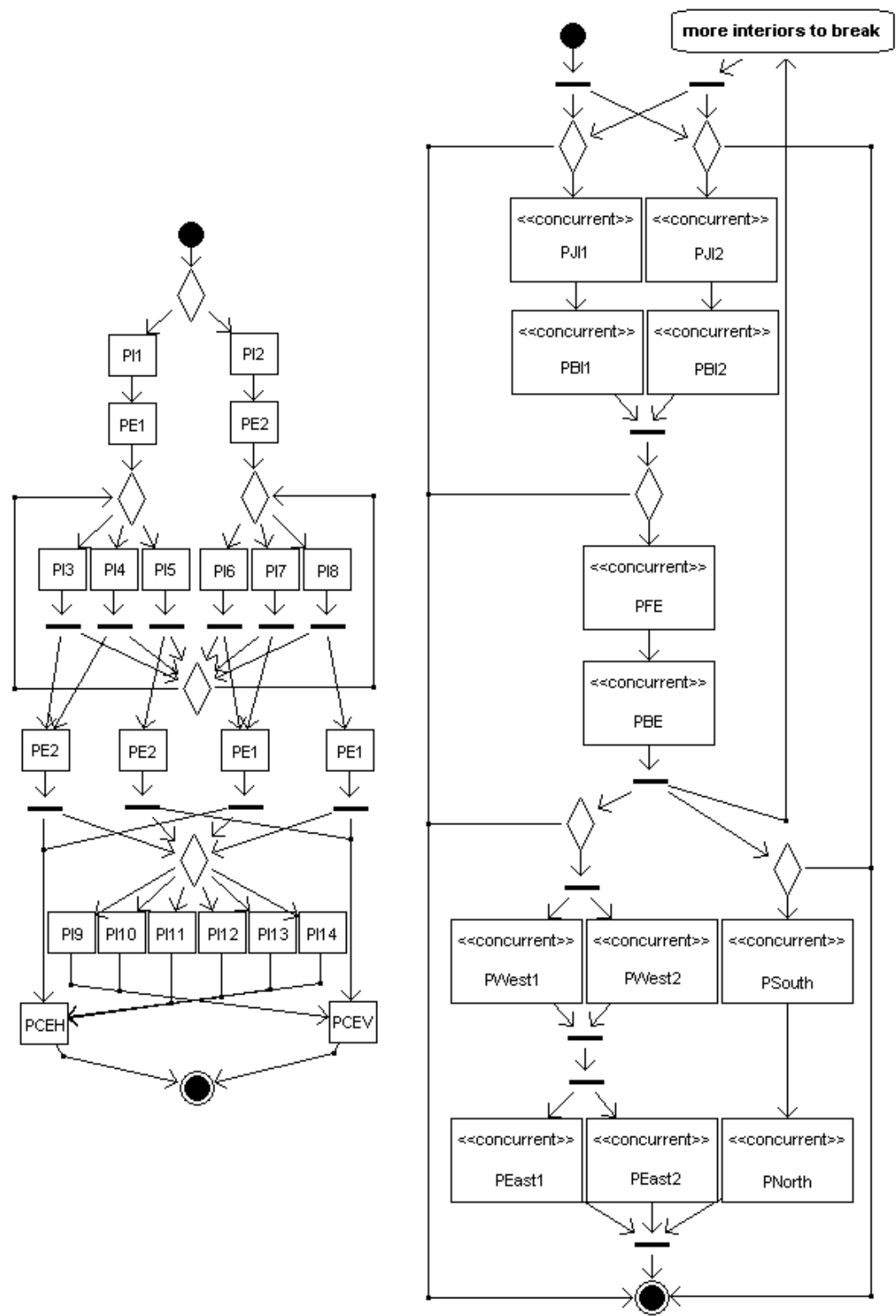

Fig. 5. Left panel: Control diagram based model of concurrency for the process of the generations of triangular finite elements two dimensional mesh. Right panel: Control diagram based model of concurrency for the process of $h$ adaptation.

the mesh elements may have to be refined in portions (enforced by the mesh regularity rule), a new portion of refinements can be started while adjacency propagation of the previous portion is still running. 


\section{Petri Nets Model of Mesh Transformations}

In this section we introduce the Petri net based model of concurrency for the initial mesh generation as well as for $h$ adaptation. In addition to the control diagram based model it provides analysis tools typical for Petri nets like reachability graphs, place/transition invariants, etc. A system modeled as a Petri net can be analyzed for deadlocks, starvation or infinite execution. This model also allows to express some quantitative relationships between graph grammar productions.

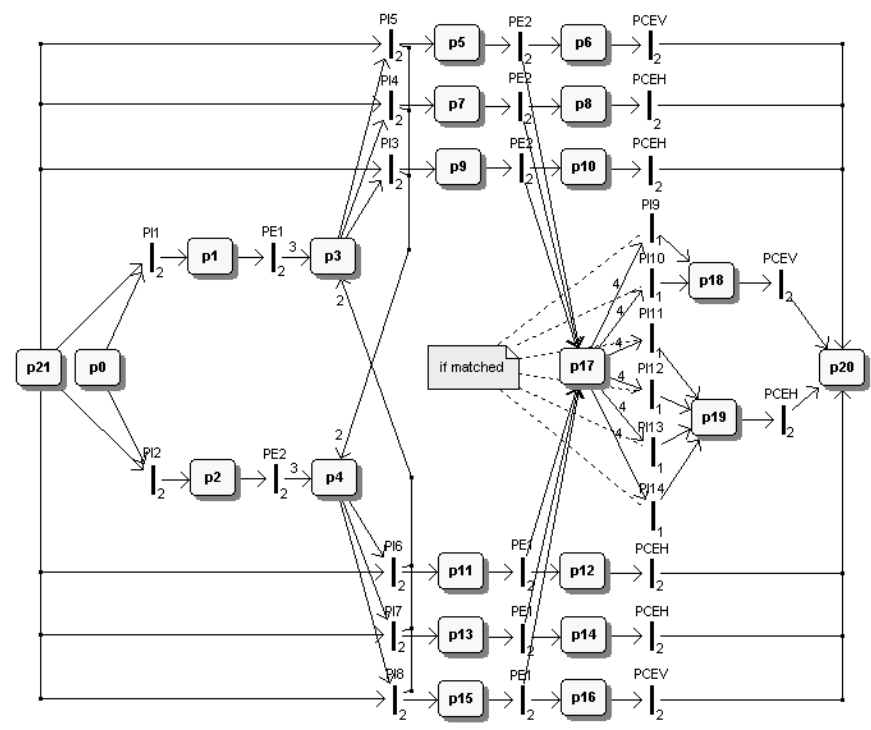

Fig. 6. Petri nets model of concurrency for the process of triangular finite element two dimensional mesh generation

Figure 6 presents a priority Petri net with guards for initial mesh generation. Transitions are named after productions they execute. Priorities are assigned to transitions (in subscript) to enforce certain sequence of execution. Transitions PI9 - PI14 (priority 1) should be executed as soon as they get activated, before any other transition being active at the same time. The prioritization has been introduced to ensure correct mesh construction (with no overlapping elements). Transitions PI9 - PI14 have an additional activation guard - they can be activated only if there is an actual matching for these productions in the mesh being generated. The condition expressed with the guard is not enforced by the net itself. Edge weights denote the number by which the marking of the destination place is increased or the marking of the origin place is decreased. The meaning of some of the places is as follows: 
- p3 number of type 2 elements that can be produced from current mesh shape

- p4 number of type 1 elements that can be produced from current mesh shape

- p21 number of elements left to be produced

The initial marking is: p0 - 1, p21 - N, where $\mathrm{N}$ is a parameter to the system telling how many elements the initial mesh will consist of. The rest of the places are empty. Just one token in place p0 implies that only one of the starting transitions (PI1 or PI2) may be fired since they are in conflict. The only transitions that increase the number of tokens in the net are PE1, PE2 and PI3 - PI8. Only one of the transitions: PE1 input to place p3 or PE2 input to place p4 may be fired and it may be fired only once due to the starting transitions conflict. Transitions PI3 - PI8 have place p21 as input which limits the number of their activations since the initial marking of that place is only decreasing during the system execution. Therefore the Petri net is bounded and the initial mesh generation will eventually stop.

The reachability graph is finite and suitable for analysis. Place p21 is the determinant of the net's liveness. When that place gets empty, the net quickly reaches a dead marking - as soon as places p5, p16 and p18 also get empty. Such a dead marking means the end of initial mesh generation; no deadlock is possible while place p21 contains tokens. The Petri net structure is fixed; different shapes of generated meshes will result in choosing different paths in the reachability graph.

Figure 7 presents the Petri net for $h$ adaptation. The initial marking of place p0 is the number of the mesh elements chosen for adaptation by an external

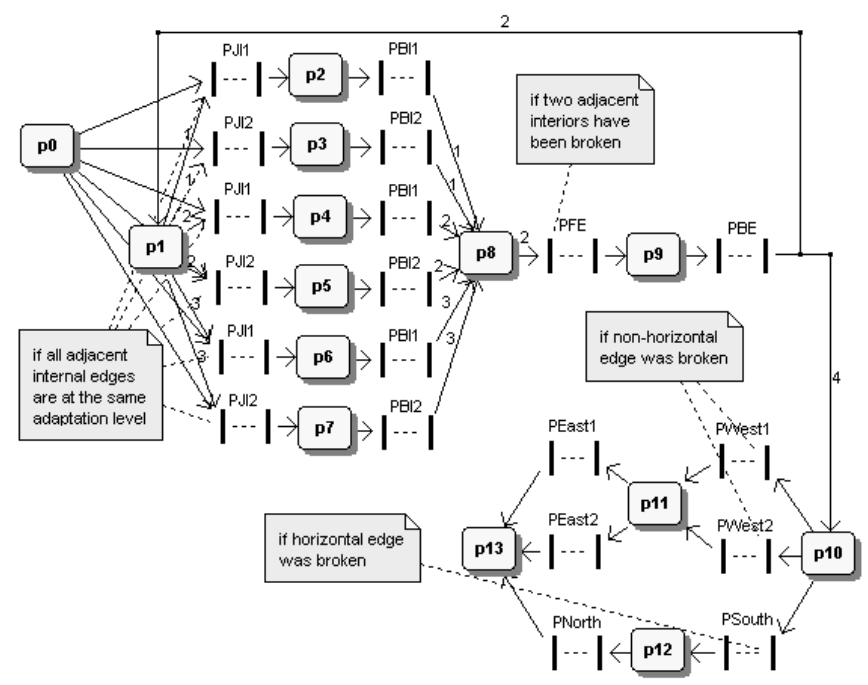

Fig. 7. Petri nets with guards model of concurrency for the process of $h$ adaptation 
driver. This includes elements that have to be broken as prerequisites to the adaptation of the chosen elements (according to the mesh regularity rule). The marking of place p1 determines the adaptation "potential" of the mesh and initially it will be: number of elements with 3 neighbors multiplied by $3+$ number of elements with 2 neighbors times $2+$ number of elements with 1 neighbor. The marking of places p1 and p8 should be preserved between subsequent iterations of $h$ adaptation.

The branch with places p2 and p3 models interior breaking of elements with 1 neighbor; branch with places p4 and p5 - elements with 2 neighbors; branch with places p6 and p7 - elements with 3 neighbors. Two transitions linked with a dashed line denote actually all activated transitions of a given type fired concurrently. Although their numbers depend on the actual problem being solved, the reachability graph is still finite and can be analyzed since the net is bounded thanks to the place p0. Mesh regularity rules are enforced with the transition guards. Therefore the net can reach a dead state even if places p1 and/or p8 still contain tokens.

\section{Conclusions}

In this paper, we presented an extended graph grammar model, dedicated for the self-adaptive $h p$-FEM algorithm with an arbitrary initial regular triangular mesh. We analyzed both algorithms for an initial mesh generation and mesh adaptation. We created the model of concurrency, based on control diagrams defining the relations between graph grammar productions. The Petri nets were created, modeling both initial mesh generation and adaptation process. This will provide the analysis tools typical for Petri nets like reachability graphs, place/transition invariants, etc. The Petri nets model of the system can be analyzed for deadlocks, starvation or infinite execution.

The future work in this field will include the analysis of concurrency potential of the remaining steps of $h p$-FEM. It may also be beneficial to employ other concurrency modeling tools for expressiveness comparison.

Acknowledgments. The work reported in this paper was partially supported by Polish Ministry of Scientific Research and Information Technology, and by the Foundation for Polish science under Homming program.

\section{References}

1. Grabska, E.: Theoretical Concepts of Graphical Modeling. Part One: Realization of CP-Graphs. Machine Graphics and Vision 2(1), 3-38 (1993)

2. Grabska, E.: Theoretical Concepts of Graphical Modeling. Part Two: CP-Graph Grammars and Languages. Machine Graphics and Vision 2(2), 149-178 (1993)

3. Grabska, E., Hliniak, G.: Structural Aspects of CP-Graph Languages. Schedae Informaticae 5, 81-100 (1993) 
4. Demkowicz, L.: Computing with hp-Adaptive Finite Elements. Chapman \& Hall/Crc Applied Mathematics \& Nonlinear Science, vol. I (2006)

5. Demkowicz, L., Kurtz, J., Pardo, D., Paszynski, M., Rachowicz, W., Zdunek, A.: Computing with hp-Adaptive Finite Elements. Chapman \& Hall/Crc Applied Mathematics \& Nonlinear Science, vol. II (2007)

6. Paszyński, M., Kurtz, J., Demkowicz, L.: Parallel Fully Automatic hp-Adaptive 2D Finite Element Package. Computer Methods in Applied Mechanics and Engineering 195(7-8)(25), 711-741 (2006)

7. Paszyński, M., Demkowicz, L.: Parallel Fully Automatic hp-Adaptive 3D Finite Element Package. Engineering with Computers 22(3-4), 255-276 (2006)

8. Paszyński, M., Paszyńska, A.: Graph transformations for modeling parallel $h p$ adaptive Finite Element Method. In: Wyrzykowski, R., Dongarra, J., Karczewski, K., Wasniewski, J. (eds.) PPAM 2007. LNCS, vol. 4967, pp. 1313-1322. Springer, Heidelberg (2008)

9. Paszyńska, A., Paszyński, M., Grabska, E.: Graph transformations for modeling $h p$ adaptive Finite Element Method with triangular elements. In: Bubak, M., van Albada, G.D., Dongarra, J., Sloot, P.M.A. (eds.) ICCS 2008, Part III. LNCS, vol. 5103, pp. 604-613. Springer, Heidelberg (2008) 